Janet Oyebode ${ }^{7}$, Jackie Pool ${ }^{8}$, Bob Woods ${ }^{9},{ }^{1}$ University of Exeter, Exeter, United Kingdom; ${ }^{2}$ Cardiff University, Cardiff, United Kingdom; ${ }^{3}$ Research Institute for the Care of Older People, Bath, United Kingdom; ${ }^{4}$ London School of Economics and Political Science, London, United Kingdom; ${ }^{5}$ Kings College London, London, United Kingdom; ${ }^{6}$ University of Manchester, Manchester, United Kingdom, ${ }^{7}$ University of Bradford, Bradford, United Kingdom; ${ }^{8}$ Dementia Pal Ltd, Southampton, United Kingdom; ${ }^{9}$ Institute of Medical and Social Care Research, Bangor University, Bangor, United Kingdom. Contact e-mail: l.clare@exeter.ac.uk

Background:Cognitive rehabilitation (CR) is an individualised, person-centred intervention for people with early-stage dementia that aims to support everyday functioning and independence. Realistic and personally-meaningful goals are agreed in collaboration with the therapist, and a plan is developed to support goal attainment using evidence-based rehabilitation strategies. Carers are involved where possible. Feasibility studies and a pilot randomised controlled trial (RCT) previously yielded positive results, corroborated by other recent evidence. Methods: GREAT was a multi-centre RCT conducted in 8 sites throughout England and Wales. Participants had an ICD-10 diagnosis of Alzheimer's, vascular or mixed dementia, were in the early stages (MMSE score $\geq 18$ ), were stable on medication if prescribed, and had a family carer willing to contribute. Exclusion criteria were a history of brain injury or other neurological disorder, and inability to speak English. Following goal-setting to identify areas of everyday functioning that could be improved or managed better, participants were randomised to receive either treatment as usual (TAU) or CR. CR consisted of 10 therapy sessions over 3 months followed by 4 maintenance sessions over 6 months, and was delivered in participants' homes by 9 occupational therapists and 1 nurse. Participants were followed up 3 months and 9 months post-randomisation. The primary outcome was self-reported and carer-reported goal attainment at 3-month follow-up. Secondary outcomes were participant quality of life, mood, self-efficacy and cognition, and carer stress, health status and quality of life. To achieve adequate power, it was necessary for 350 people to complete the trial, 175 in each arm. Results: 475 participants were randomised, of whom 427 completed the trial ( $C R \mathrm{n}=209$, TAU $\mathrm{n}=218$ ). At 3-month follow-up there were statistically-significant large positive effects for participant-rated goal attainment $(\mathrm{d}=.97,95 \% \mathrm{CI} .75-1.19)$, corroborated by carer ratings $(\mathrm{d}=1.11, .89-1.34)$. These effects were maintained at 9-month follow-up for both participant $(\mathrm{d}=.94, .71-1.17)$ and carer ratings $(d=.96, .73-1.2)$. There were no significant differences in secondary outcomes. Conclusions: Cognitive rehabilitation is effective in supporting everyday functioning for people with early-stage Alzheimer's, vascular or mixed dementia, and could form a valuable component of post-diagnostic care pathways and community reablement or home care packages.

\section{O3-02-06 TRANSFER EFFECT AFTER MNEMONIC STRATEGY TRAINING IN AMNESTIC MILD COGNITIVE IMPAIRMENT: EVIDENCE FROM A RANDOMIZED, SINGLE-BLIND STUDY}

Sharon Sanz Simon ${ }^{1}$, Benjamin M. Hampstead ${ }^{2}$, Cassio Machado de Campos Bottino ${ }^{3}$, Fabio Henrique de Gobbi Porto ${ }^{4}$, Fabio L. S. Duran ${ }^{5}$, Sonia Maria Dozzi Brucki ${ }^{4}$, Camila B. Martins ${ }^{6}$, Renata Ávila ${ }^{7}$, Geraldo Busatto Filho ${ }^{8}$, Edson amaro, Jr. ${ }^{1}$, Mariana P. N. Silva ${ }^{1}$, Luciana M. Fonseca ${ }^{1}$, Maria da Graça M. Martins ${ }^{1}$,
Lyssandra dos Santos Tascone ${ }^{9},{ }^{1}$ University of São Paulo School of Medicine, São Paulo, Brazil; ${ }^{2}$ University of Michigan, Ann Arbor, MI, USA, ${ }^{3}$ Old Age Research Group (PROTER), Faculty of Medicine, University of São Paulo, São Paulo, Brazil; ${ }^{4}$ Medical School of University of São Paulo, São Paulo, Brazil, ${ }^{5}$ University of Sao Paulo, School of Medicine, Sao Paulo, Brazil; ${ }^{6}$ Federal University of São Paulo / Paulsita School of Medicine, São Paulo, Brazil; ${ }^{7}$ University of Sao Paulo/School of Medicine, São Paulo, Brazil; ${ }^{8}$ University of São Paulo/ School of Medicine, São Paulo, Brazil; ${ }^{9}$ University of São Paulo / Medical School, São Paulo, Brazil.

Contact e-mail: sharon.sanzsimon@gmail.com

Background: Evidence suggests benefits of mnemonic strategy training (MST) in Mild Cognitive Impairment (MCI), although it remains unclear the transfer effect and neurobiology underlying this intervention. Face-name association training is an ecologically relevant target of MST since forgetting names is a frequent complaint in elders with MCI, with possible impact in social life. Methods: Thirty participants were randomized to MST for face-name associations or education program (EP), an active control condition. All patients completed four individual one-hour sessions, twice a week. At baseline, participants underwent clinical and neuropsychological assessments as well as, CSF and MRI exams. During fMRI, the subjects performed a face-name encoding task and then completed the Face-Name Recognition Task (FNRT) outside the scan. One-week after the programs, patients repeated the fMRI, the FNRT, neuropsychological tests (HVLT-R and SKT), Strategy Use Task (SUT), metamemory questionnaire (MMQ) and mood scales (BAI and BDI). The cognitive and behavioral measures were repeated at one and threemonth follow-ups. Results: Groups were comparable at baseline. Regarding the FNRT, relative to EP, MST group showed a trend toward better accuracy, significant increase of confidence level and slower performance on untrained stimuli, a finding that suggests the use of MST. Also, MST significantly improved the memory performance on SKT, increased the use of an associative strategy (SUT), however no changes were observed on HVLT-R. Moreover, MST participants reported less memory mistakes in everyday life (MMQ-mistakes), and no changes on mood inventories. Neuroimaging findings indicate that after the programs MST group showed greater brain activation than EP participants in the left anterior and lateral temporal cortices (e.g., superior and middle temporal gyrus). Conclusions: MST transfer to untrained stimuli, and, in part, to neuropsychological tests. Moreover, relative to EP, after MST it was observed a greater use of an associative strategy, and a more positive perception of the memory abilities. Moreover, the MST groups showed a greater increase of brain activation than ED in regions relevant to process of faces and social cues, perception of physical features and emotions, semantic memory and associative thinking.

\section{TUESDAY, JULY 18, 2017 ORAL SESSION \\ O3-03 \\ NEUROIMAGING: TAU AND \\ NEURODEGENERATION-DEMENTIA SYNDROMES}

\section{3-03-01 FLORTAUCIPIR TAU-PET SPECIFICITY IS MAINTAINED IN PATIENTS WITH PATHOLOGICALLY CONFIRMED CREUTZFELDT-JAKOB DISEASE}

Gregory S. Day ${ }^{1,2}$, Brian A. Gordon ${ }^{3,4}$, Richard J. Perrin ${ }^{3,5}$, Nigel J. Cairns ${ }^{3,4}$, Katherine E. Schwetye ${ }^{3}$, Cole J. Ferguson ${ }^{3}$, 